\title{
Diplopia After Maxillary Nerve Block Through the Lateral Infrazygomatic Approach: A Case Report and Literature Review
}

This article was published in the following Dove Press journal:

Journal of Pain Research

\author{
Minkyung Kim (D) \\ Yoomin $\mathrm{Oh}^{1}$ \\ Jeongsoo $\operatorname{Kim} \mathbb{( D}^{1,2}$ \\ 'Department of Anesthesiology and Pain \\ Medicine, Seoul National University \\ Hospital, Seoul, Republic of Korea; \\ ${ }^{2}$ Department of Anesthesiology and Pain \\ Medicine, SMG-SNU Boramae Medical \\ Center, Seoul, Republic of Korea
}

\begin{abstract}
Maxillary nerve block is widely used for treating trigeminal neuralgia. Common complications of the procedure include bleeding and sensory abnormalities, but ophthalmic complications have been rarely reported. A 60-year-old woman underwent maxillary nerve block for refractory trigeminal neuralgia. Ten minutes after the procedure had ended, the patient reported double vision when she tried to turn the left eye outward. After examination, the patient was presumed to have diplopia due to the abducens nerve block. However, the symptom disappeared approximately 30 minutes later without any treatment. Thus, to prevent diplopia, the dose of local anesthetics should be reduced. Moreover, negative blood aspiration should be confirmed during the injection. To the best of our knowledge, this is the first case to report diplopia after maxillary nerve block without neurolysis through the lateral infrazygomatic approach.
\end{abstract}

Keywords: diplopia, nerve block, trigeminal neuralgia

\section{Introduction}

Trigeminal neuralgia is one of the typical diseases that lead to neuropathic facial pain. Blockade of the trigeminal nerve branch in the affected area is a common treatment procedure. Bleeding and some sensory abnormalities are common side effects of trigeminal nerve block. In contrast, the incidence of ophthalmic complications is relatively low. ${ }^{1}$ Chavali et al reported the case of a patient with trigeminal neuralgia, in whom alcohol neurolysis of the maxillary nerve through the lateral infrazygomatic approach caused diplopia. ${ }^{2}$ A few other papers reported diplopia occurring during trigeminal radiofrequency thermocoagulation or alcohol neurolysis with the approach through the foramen ovale. ${ }^{3-5}$ However, to the best of our knowledge, there have been no reports of diplopia after maxillary nerve block without neurolysis achieved through the lateral infrazygomatic approach. In this paper, we report a rare case of temporary diplopia that occurred after maxillary nerve block performed through the lateral infrazygomatic approach in a patient with trigeminal neuralgia, with a literature review of other diplopia cases after neurolysis or radiofrequency thermocoagulation.

\section{Case Presentation}

A 60-year-old female patient diagnosed with trigeminal neuralgia involving the left maxillary nerve had a severe pain score of 8 on the 11-point numerical rating scale
Correspondence: Jeongsoo Kim Department of Anesthesiology and Pain Medicine, Seoul National University Hospital, Seoul, 03080, Republic of Korea Tel +82-2-2072-2467

Email dreamsu457@gmail.com

Journal of Pain Research 202I:|4 48|-485 
(NRS). The patient had been taking carbamazepine (600 mg/ daily), celecoxib (200 mg/daily), pregabalin (200 mg/daily), and tramadol (75 mg/daily) prescribed at the SMG-SNU Boramae Medical Center since July 2019. The patient complained of somnolence during the day and refused to increase the dosage of oral medication. Therefore, maxillary nerve block was performed for symptom control. Magnetic resonance imaging (MRI) showed no specific intracranial abnormality.

After the patient was placed in the supine position and a sterile drape was placed, the needle entry site was anesthetized with $0.5 \mathrm{~mL}$ of $1 \%$ lidocaine using a hypodermic needle horizontally. An experienced pain specialist (J Kim) inserted a 22-gauge spinal needle (Taechang Industry, Gongju-si, Chungcheongnam-do, Korea) into the upper pterygopalatine fossa using the lateral infrazygomatic approach under fluoroscopic guidance. When the needle tip was placed on the upper part of the pterygopalatine fossa in the fluoroscopic lateral view, close to the perpendicular plate of the palatine bone, which is the medial boundary of the fossa in the anteroposterior view, the contrast media was injected (Figure 1). After the contrast media sufficiently filled the pterygopalatine fossa without vascular uptake and confirming negative blood aspiration, a mixture of $5 \mathrm{mg}$ dexamethasone in $1.5 \mathrm{~mL} 0.17 \%$ levobupivacaine was injected. 6,7

Approximately 10 minutes after the nerve block, the patient complained about diplopia when trying to turn her eyes outward. On examination, the patient could not move her left eye laterally (Figure 2). On bedside ophthalmological examination, her visual acuity, pupil reflex, and cornea were found to be normal. We assumed that the abducens nerve had been blocked temporarily and decided to observe the patient for a while. However, the symptom disappeared approximately 30 minutes later without any treatment. The patient was discharged on the same day without additional examination and was instructed to visit the hospital in case of recurrence.

At the outpatient follow-up a month after discharge, the patient did not complain of any ophthalmic complications; the pain severity had decreased from a score of 8 to 3 on the 11-pointed NRS and patient satisfaction was significantly higher. The patient is planning to undergo pulsed radiofrequency neuromodulation therapy.

\section{Ethics Statement}

This study was approved by the Institutional Review Board (IRB) of SMG-SNU Boramae Medical Center (IRB No. 302020-086). We received the patient's informed consent form about publishing all photographs and reports.

\section{Discussion}

Common side effects after trigeminal nerve block include sensory abnormalities, bleeding, and infection. However, ophthalmic complications are relatively rare. It occurs in approximately $0.02 \%$ of patients after alcohol neurolysis and approximately $2 \%$ after radiofrequency thermocoagulation to the trigeminal nerve. ${ }^{1,5}$ Previous cases of diplopia in patients who had undergone radiofrequency thermocoagulation and alcohol neurolysis are listed in Table 1. However, there was no previous case report of diplopia that was induced by local anesthetics alone in trigeminal nerve

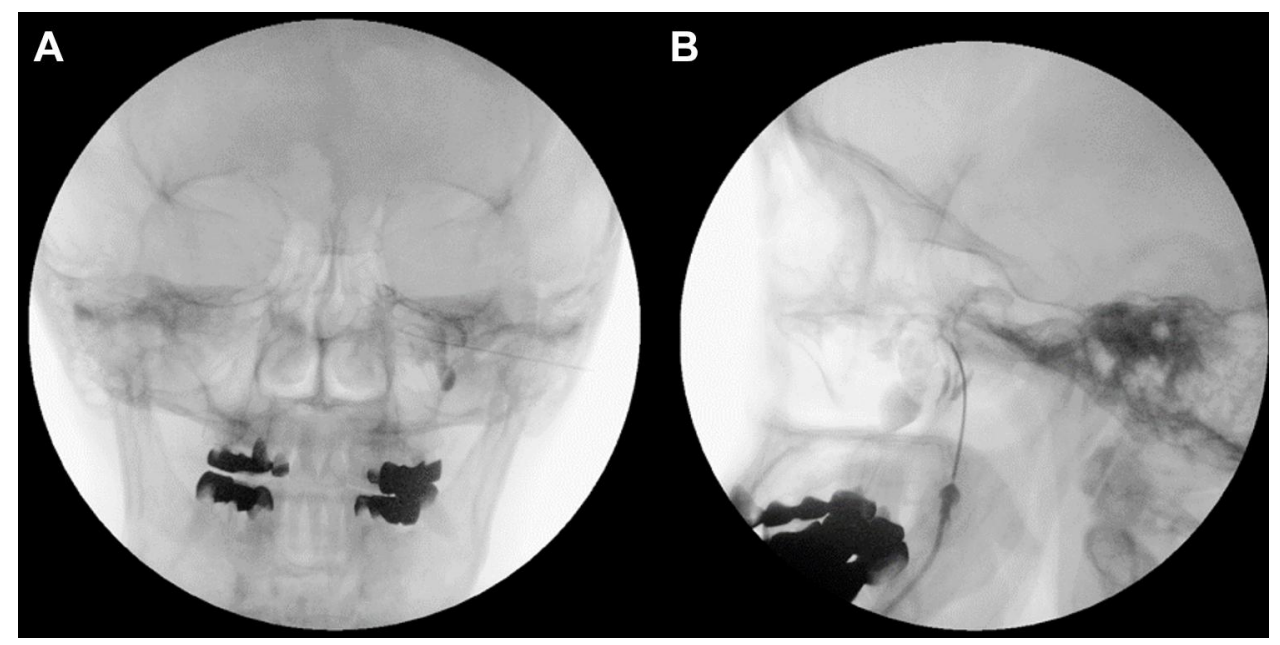

Figure I (A) Fluoroscopic anteroposterior view after contrast medium injection. (B) Fluoroscopic lateral view after contrast medium injection. Note: The contrast medium flowed into the inferior orbital fissure. 


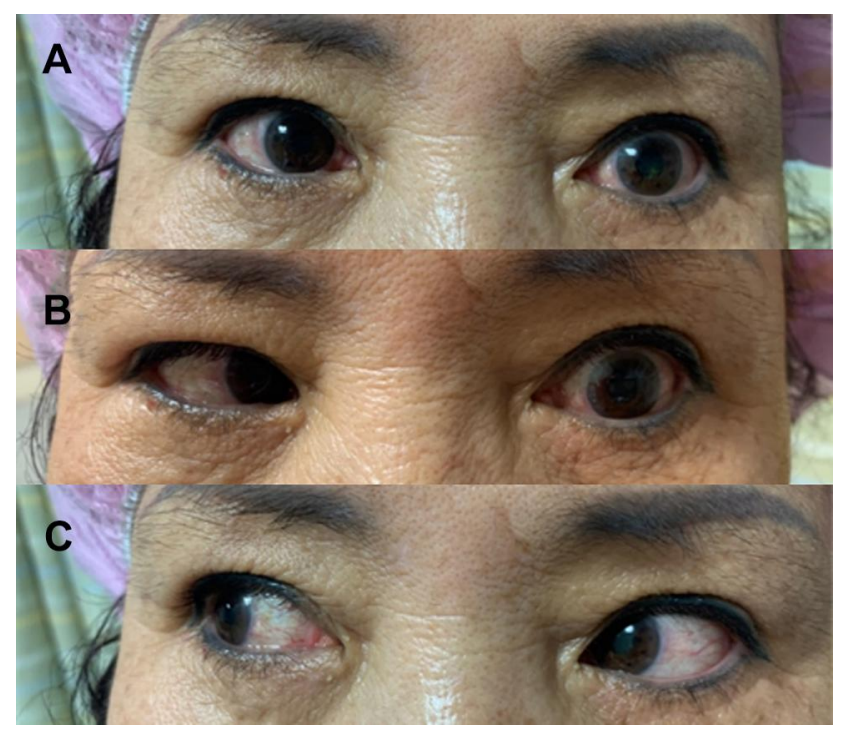

Figure 2 Clinical photos of (A) central gaze, (B) left-sided gaze, and (C) right-sided gaze with left-sided abducens nerve palsy.

Note: Left-sided gaze is not possible with the left eye.

block. Comparing with previous reports, our case shows diplopia with local anesthetics alone without alcohol neurolysis or radiofrequency thermocoagulation. Furthermore, it takes 2-6 months until complete recovery of diplopia, but in our case, the symptoms were resolved within 30 minutes. Therefore, we report a present case with literature reviews and hypotheses about the cause of diplopia.

There are three possible explanations for diplopia after maxillary nerve block under the lateral infrazygomatic approach. First, the anesthetic agent flows retrogradely from the sphenopalatine artery to the internal maxillary artery and terminates in the lacrimal and ophthalmic arteries that supply the lateral rectus muscle. ${ }^{8}$ Second, the anesthetic agent accidentally flows into the pterygoid plexus and drains into the cavernous sinus, affecting the abducens nerve, which runs through the lateral wall of the sinus. ${ }^{2}$ Lastly, direct diffusion of the anesthetic agent into the abducens nerve from the inferior to superior orbital fissure via the pterygopalatine fossa due to its proximity might cause diplopia (Figure 3). ${ }^{2,9}$ In our case, considering the contrast spread pattern in the fluoroscopic figure, diffusion was the most likely cause.

Considering the following hypotheses, we suggest several precautions to minimize ophthalmic complications. First, when performing maxillary nerve block, sensory and motor stimulation can be used to locate the nerve more accurately and reduce complications. Second, to avoid transarterial and transvenous injection, it is important to carefully check the pattern of contrast spread and negative blood aspiration. A fluoroscopic technique such as digital subtraction angiography can be utilized. Last, to prevent direct diffusion of the anesthetic agent into the adjacent nerves, it is necessary to inject the appropriate volume of the agent. Empirically, 2.5-3.0 mL of anesthetics are commonly used in maxillary nerve block; in our patient, we used $2.5 \mathrm{~mL} .{ }^{10}$ According to an anatomical study, the volume of the pterygopalatine fossa in most dried skulls was below $1 \mathrm{~cm}^{3}$; the median volume was $0.7 \mathrm{~cm}^{3}$. The empirical volume of local anesthetics injected, therefore, exceeds the volume of the pterygopalatine fossa. ${ }^{11}$ The volume remaining after filling the pterygopalatine fossa may thus diffuse into adjacent structures.

Table I Literature Review for Diplopia Following Percutaneous Procedures in Patients with Trigeminal Neuralgia

\begin{tabular}{|l|l|l|l|l|l|l|l|l|}
\hline Author & $\begin{array}{l}\text { Age } \\
\text { (Years) }\end{array}$ & Sex & Procedure & Approach & Volume & $\begin{array}{l}\text { Symptom } \\
\text { Onset }\end{array}$ & $\begin{array}{l}\text { Complete } \\
\text { Recovery } \\
\text { (Months) }\end{array}$ & $\begin{array}{l}\text { Causes Deduced from the } \\
\text { Article }\end{array}$ \\
\hline $\begin{array}{l}\text { Chavali } \\
\text { et al }\end{array}$ & 45 & Male & $\begin{array}{l}\text { Alcohol } \\
\text { neurolysis }\end{array}$ & $\begin{array}{l}\text { Lateral } \\
\text { infrazygomatic }\end{array}$ & $\begin{array}{l}\text { Alcohol } \\
\mathrm{I} \mathrm{mL}\end{array}$ & Few seconds & 2 & $\begin{array}{l}\text { Incidental intraarterial or } \\
\text { intravenous injection }\end{array}$ \\
\hline Choi et al & 35 & Female & $\begin{array}{l}\text { Alcohol } \\
\text { neurolysis }\end{array}$ & FO & $\begin{array}{l}\text { Alcohol } \\
0.5 \mathrm{~mL}\end{array}$ & $\begin{array}{l}\text { Right after } \\
\text { procedure }\end{array}$ & 3 & $\begin{array}{l}\text { Close anatomic proximity of } \\
\text { the abducens nerve to } \\
\text { trigeminal complex }\end{array}$ \\
\hline $\begin{array}{l}\text { Chatterjee } \\
\text { et al }{ }^{4}\end{array}$ & 42 & Male & RFA & FO & $\begin{array}{l}5 \text { minutes } \\
\text { after } \\
\text { procedure }\end{array}$ & 6 & $\begin{array}{l}\text { Close anatomic proximity of } \\
\text { the abducens nerve to } \\
\text { trigeminal complex }\end{array}$ \\
\hline $\begin{array}{l}\text { Harrigan } \\
\text { et al }\end{array}$ & 86 & Male & RFA & FO & $\begin{array}{l}\text { Righter } \\
\text { anesthesia }\end{array}$ & 2 & $\begin{array}{l}\text { Close anatomic proximity of } \\
\text { the abducens nerve to } \\
\text { trigeminal complex }\end{array}$ \\
\hline
\end{tabular}

Abbreviations: FO, foramen ovale; RFA, radiofrequency ablation. 


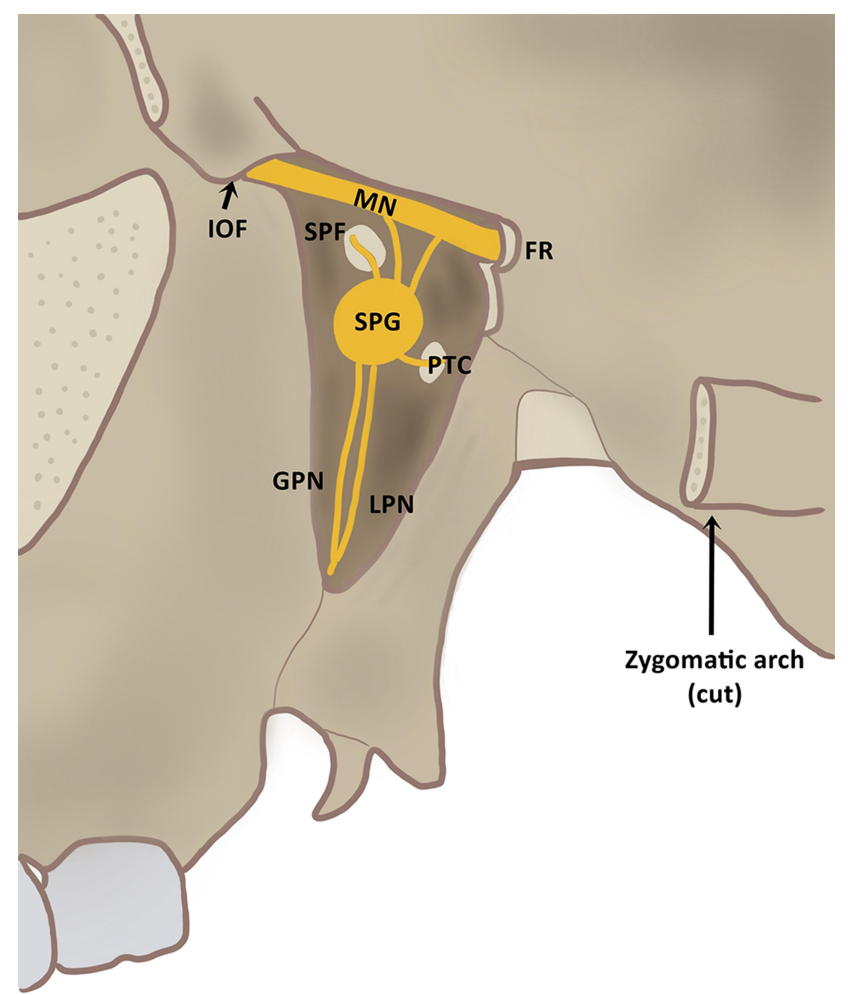

Figure 3 Schematic illustration of the pterygopalatine fossa.

Abbreviations: FR, foramen rotundum; GPN, greater palatine nerve; IOF, inferior orbital fissure; LPN, lesser palatine nerve; MN, maxillary nerve; PTC, pterygoid canal; SPF, sphenopalatine foramen; SPG, sphenopalatine ganglion.

Empirical volumes of anesthetic agents can cause ophthalmic complications. Therefore, we suggest smaller volumes of local anesthetics for preventing side effects, such as the diplopia that occurred in our case. Further study of the ideal volume less than empirical volumes is needed for minimization of complications.

According to previous studies in dentistry, the incidence of ophthalmic complications, including diplopia, after maxillary nerve block for dental anesthesia is relatively high. Sved et al reported that $35 \%$ of patients developed diplopia after maxillary nerve block through the greater palatine canal. ${ }^{10}$ Maxillary nerve block for dental anesthesia is performed without ultrasound or fluoroscopy. However, it is routinely performed under fluoroscopic guidance for interventional pain management, such as when using the lateral infrazygomatic approach. Therefore, accurate positioning of the needle can be confirmed; this may contribute to reduced diplopia or other ophthalmic complications. Lastly, the injection needle points toward the cephalad side in dental anesthesia but is relatively horizontal in trigeminal neuralgia treatment. There are two approach techniques to achieve maxillary nerve block in dental anesthesia: the high tuberosity approach and the greater palatine canal approach. ${ }^{12}$ In the high tuberosity approach, the needle insertion angle is approximately 45 degrees with the transverse plane of the head. ${ }^{12}$ In the greater palatine canal approach, the median optimal angle of inserting the needle into the greater palatine foramen is suggested to be 45.88 degrees. ${ }^{13}$ However, in a study with 6 patients who underwent the lateral infrazygomatic approach, the optimal mean needle insertion angle with the transverse plane was suggested to be 27.7 degrees. ${ }^{14}$ Therefore, the injectate may flow more vertically during dental anesthesia. Considering the anatomical 3-dimensional image, it is more likely to spread to the inferior orbital fissure, subsequently spreading to the superior orbital fissure, through which the abducens nerve passes (Figure 4).

In conclusion, we report a case of diplopia after maxillary nerve block in trigeminal neuralgia with a literature

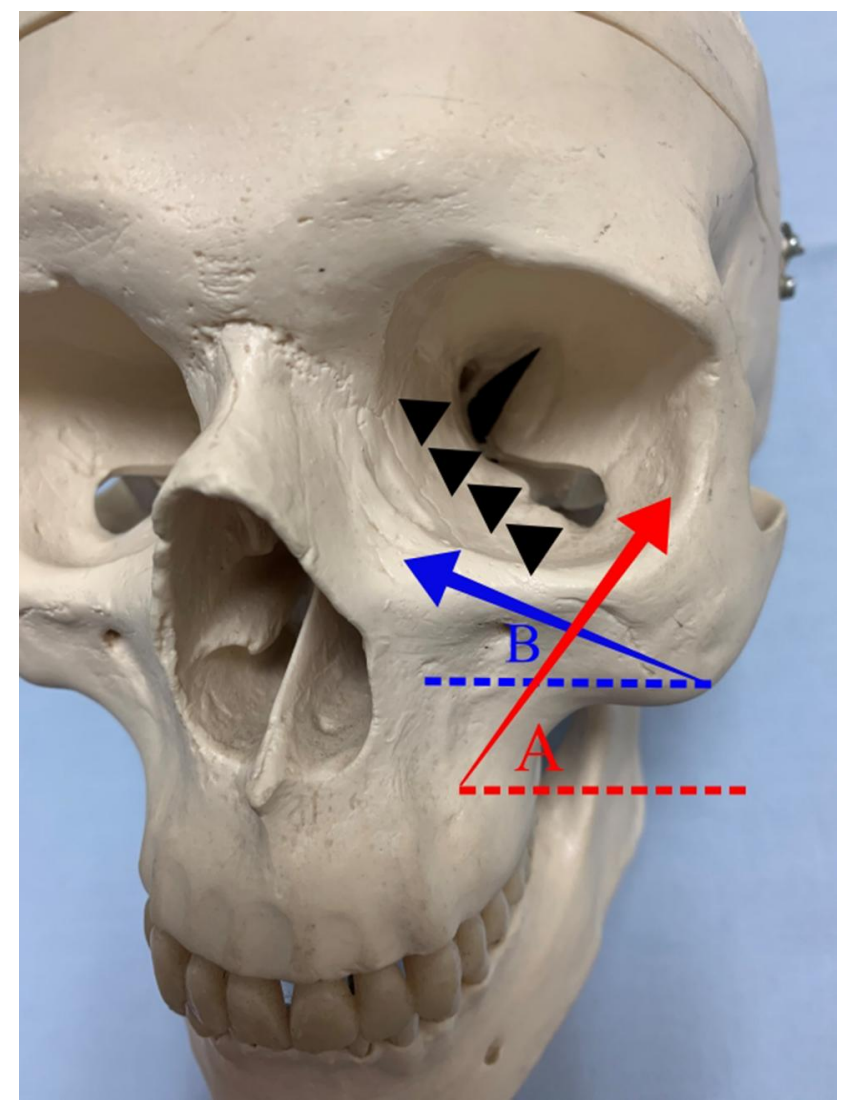

Figure 4 Schematic anatomical pathways of the maxillary nerve block. Maxillary nerve block through the intraoral approach for dental anesthesia (red arrow, $\mathrm{A}=$ approximately 45 degrees) and the lateral infrazygomatic approach for trigeminal neuralgia treatment (blue arrow, B = approximately 27.7 degrees). The orbital fissure (black triangles) through which the abducens nerve passes.

Note: During maxillary nerve block for dental anesthesia, the injectant may flow better into the inferior orbital fissure at the top of the pterygopalatine fossa because the injectant is injected more vertically. 
review of similar cases. To the best of our knowledge, this is the first case report of diplopia after maxillary nerve block in trigeminal neuralgia treatment with local anesthetics alone, not by radiofrequency thermocoagulation or alcohol neurolysis through the lateral infrazygomatic approach. We believe it is important to use the appropriate amount of injectate and to confirm negative blood aspiration.

\section{Acknowledgments}

We would like to thank Editage for English language editing.

\section{Disclosure}

The authors received no financial support for the research, authorship, and/or publication of this article. The authors have no potential conflicts of interest to disclose.

\section{References}

1. Han KR, Kim C, Kim DW, et al. Long-term outcome of trigeminal nerve block with alcohol for the treatment of trigeminal neuralgia. Korean J Pain. 2006;19:45-50. doi:10.3344/kjp.2006.19.1.45

2. Chavali S, Rath GP, Bithal PK. Diplopia following extraoral maxillary nerve blockade for trigeminal neuralgia. $J$ Neuroanaesth Crit Care. 2018;5:193-194. doi:10.1055/s-0038-1665546

3. Choi AY, Chung SA, Yun IS. A case of abducens nerve palsy after percutaneous nerve block for trigeminal neuralgia. $J$ Korean Ophthalmol Soc. 2013;54:524-528. doi:10.3341/jkos.2013.54.3.524
4. Chatterjee N, Chatterjee S, Roy C. Abducens nerve palsy after percutaneous radiofrequency ablation of gasserian ganglion. $J$ Neurosurg Anesthesiol. 2014;26:89-90. doi:10.1097/ANA.0b 013e31829ddeed

5. Harrigan MR, Chandler WF. Abducens nerve palsy after radiofrequency rhizolysis for trigeminal neuralgia: case report. Neurosurgery. 1998;43:623-625. doi:10.1097/00006123-199809000-00134

6. Dach F, Éckeli ÁL, Ferreira Kdos S, Speciali JG. Nerve block for the treatment of headaches and cranial neuralgias - a practical approach. Headache. 2015;55:59-71. doi:10.1111/head.12516

7. Nader A, Kendall MC, De Oliveria GS, et al. Ultrasound-guided trigeminal nerve block via the pterygopalatine fossa: an effective treatment for trigeminal neuralgia and atypical facial pain. Pain Physician. 2013;16(5):E537-E545.

8. Saddawi-Konefka R, Jafari A, Bracken D, et al. Transient abducens nerve palsy after sphenopalatine block following endoscopic sinus surgery. JAMA Otolaryngol Head Neck Surg. 2019;145:871-873. doi:10.1001/jamaoto.2019.1875

9. Pragasm M, Managutti A. Diplopia with local anesthesia. Natl J Maxillofac Surg. 2011;2:82-85. doi:10.4103/0975-5950.85861

10. Sved AM, Wong JD, Donkor P, et al. Complications associated with maxillary nerve block anaesthesia via the greater palatine canal. Aust Dent J. 1992;37:340-345. doi:10.1111/j.1834-7819.1992.tb00758.x

11. Stojcev Stajcić L, Gacić B, Popović N, et al. Anatomical study of the pterygopalatine fossa pertinent to the maxillary nerve block at the foramen rotundum. Int J Oral Maxillofac Surg. 2010;39:493-496. doi:10.1016/j.ijom.2009.11.002

12. Bacci C, Ferrario S, Sivolella S, et al. Maxillary nerve block: a comparison between the greater palatine canal and high tuberosity approaches. DM Ital J Dent Med. 2018;3:50-59.

13. Malamed SF, Trieger N. Intraoral maxillary nerve block: an anatomical and clinical study. Anesth Prog. 1983;30:44-48.

14. Taira Y, Higa M, Iso A, et al. A method of radiographic guiding for maxillary nerve block (pterygopalatine fossa oblique view). $J$ Anesth. 1998;12:212-214. doi:10.1007/BF02481733
Journal of Pain Research

\section{Publish your work in this journal}

The Journal of Pain Research is an international, peer reviewed, open access, online journal that welcomes laboratory and clinical findings in the fields of pain research and the prevention and management of pain. Original research, reviews, symposium reports, hypothesis formation and commentaries are all considered for publication. The manuscript management system is completely online and includes a very quick and fair peer-review system, which is all easy to use. Visit http:// www.dovepress.com/testimonials.php to read real quotes from published authors. 